\title{
MiniBooNE Neutrino Cross Section Measurements
}

\author{
Geoffrey B. Mills ${ }^{1}$ \\ Los Alamos National Laboratory \\ Los Alamos, NM, USA \\ E-mail: millselanl.gov \\ For the MiniBooNE Collaboration
}

The MiniBooNE experiment has operated at Fermilab since 2002. It has collected $6.46 \times 10^{20}$ pot in neutrino mode and $5.66 \times 10^{20}$ pot in antineutrino mode. This paper presents some of the cross section measurements from this data. The three neutrino-nucleus channels discussed here are important channels for current and future neutrino oscillation experiments.

35th International Conference of High Energy Physics (ICHEP2010)

Paris,France

July 22-28, 2010

Speaker 


\section{MiniBooNE Cross Section Measurements}

The MiniBooNE detector is described elsewhere [1]. This paper presents cross section measurements [2] made with the MiniBooNE detector. The cross sections are important for the understanding of neutrino interactions in neutrino oscillation experiments. Events in MiniBooNE are reconstructed by using a likelihood technique, originally developed for LSND and expanded for MiniBooNE events [3]. The neutrino flux was constrained by measurements of pion production in proton-beryllium interactions by the HARP collaboration [4].

\subsection{The Charged Current Quasi Elastic (CCQE) Channel}

Figure 1-1 shows the total CCQE cross section as a function of measured neutrino energy. The data is substantially above the independent particle nuclear model (dashed line), The solid line corresponds to fitting and effective axial mass $\left(M_{A}^{\text {eff }}\right)$ and minimum Fermi energy scale factor ( $\kappa)$ to the data. The RPA corrections of [5] that include 2-body currents also seem to reproduce the data well. The CCQE events are defined as any $\mathrm{CC}$ event that has no observed pion in the final state. There is a substantial single pion background that is be estimated and subtracted from the CCQE sample.

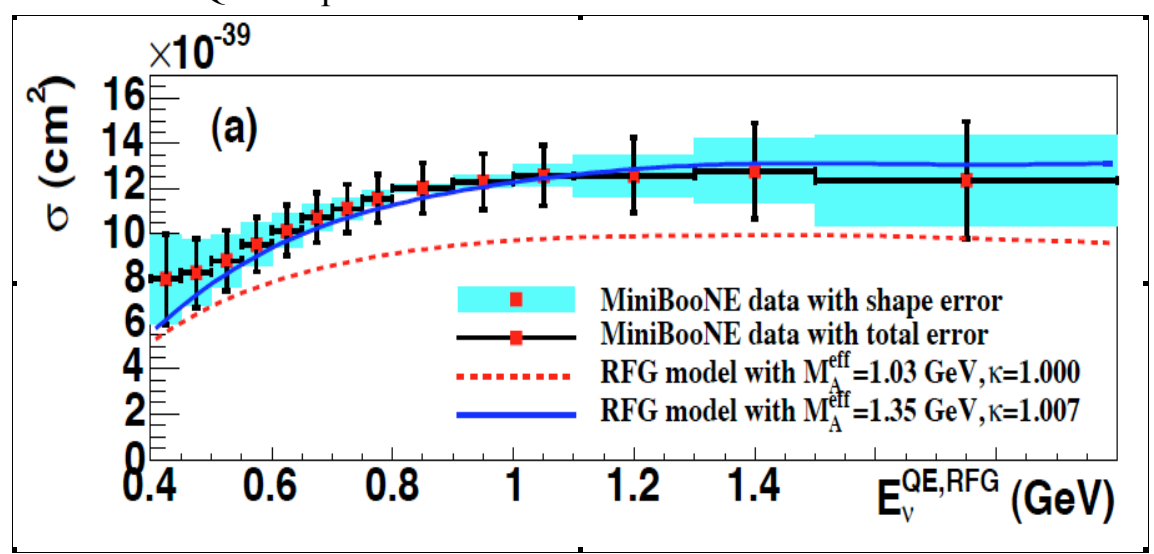

Figure 1-1 The measured CCQE cross section versus neutrino energy. The dashed curve is the standard independent particle model prediction while the red curve shows the prediction when n-particle - n-hole $R P A$ corrections are included.

\subsection{The Charged Current Single $\pi^{+}$and $\pi^{0}\left(C C \pi^{+}\left(\pi^{0}\right)\right)$ Channel}

Figure 1-2 shows the measured cross sections for single $\pi^{+}$and $\pi^{0}$ events versus neutrino energy. Those measurements required the development of extended fitting algorithms in order to reconstruct the additional pion in the event.

\subsection{The Neutral Current Quasi Elastic (NCE) and Single $\pi 0$ (NC $\pi 0)$ Channels}

Figure 1-3 shows the flux averaged cross sections for: neutral current quasi elastic versus $\mathrm{Q}^{2}$; and for neutral current single $\pi^{0}$ events versus $\pi^{0}$ momentum and angle for neutrino mode and antineutrino mode. 

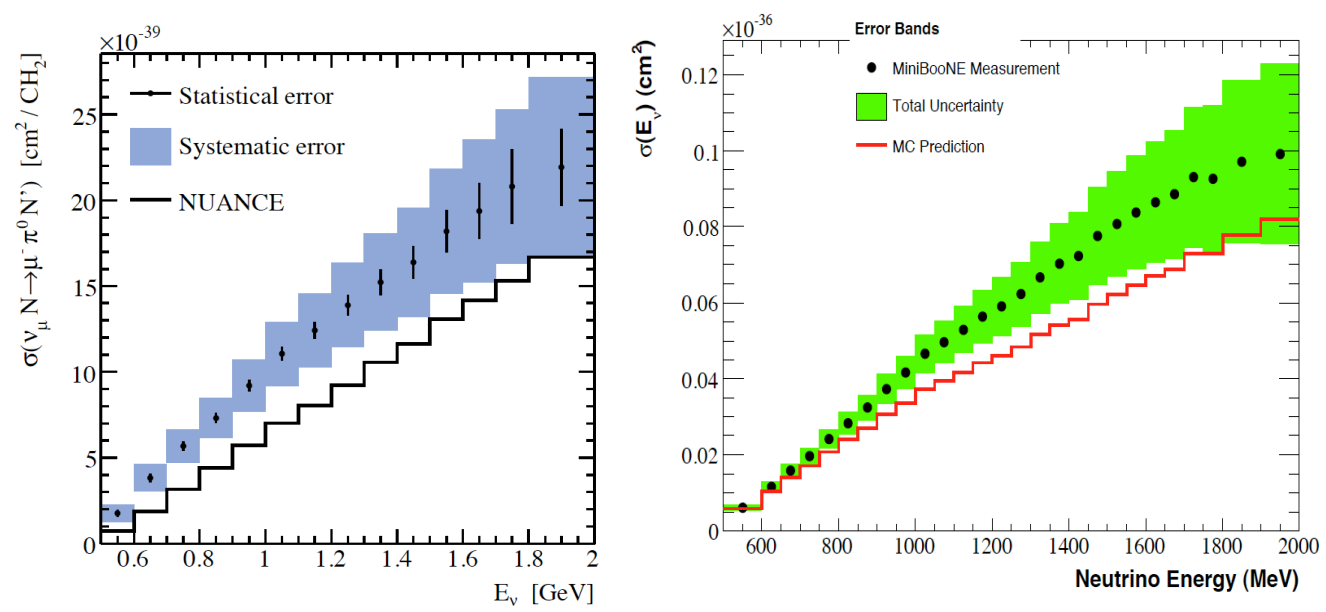

Figure 1-2 The charged current, single $\pi^{0}$ (left) and single $\pi^{+}$(right) cross sections measured by MiniBooNE are shown. The solid lines are predictions by NUANCE.
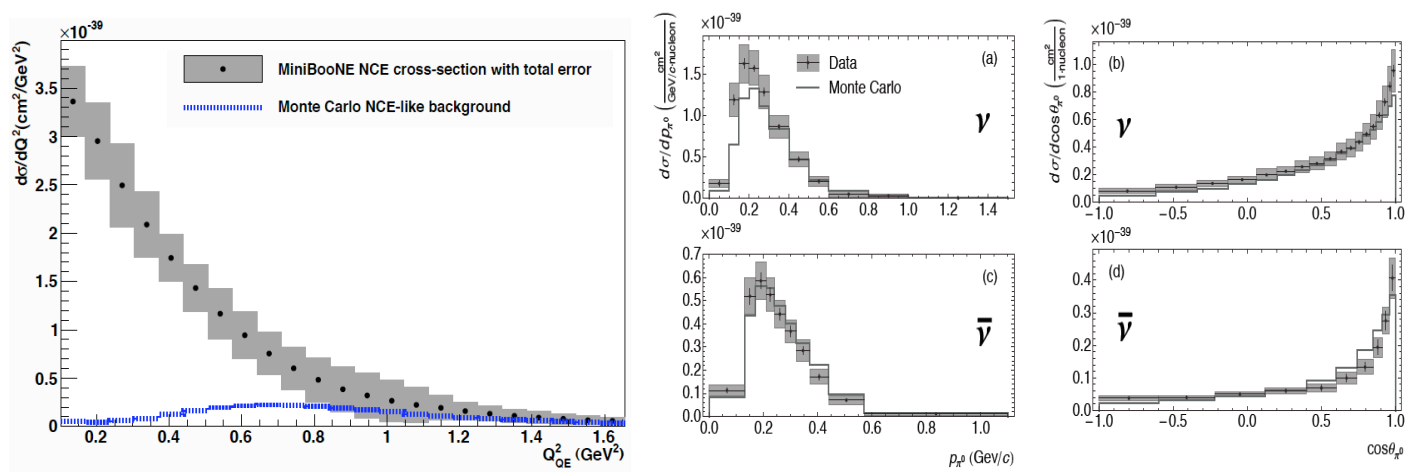

Figure 1-3 The right-hand plot shows the measured cross section for neutral current quasi elastic events versus $Q^{2}$, averaged over the MiniBooNE flux. The four panels on the right show the neutral current cross sections for single $\pi^{0}$ production versus $\pi^{0}$ momentum (middle) and $\pi^{0}$ angle (left) for neutrino mode (top) and antineutrino mode (bottom), averaged over the MiniBooNE flux.

\section{References}

[1] A. A. Aguilar-Arevalo et al. [The MiniBooNE Collaboration], NIM A599, 28-46 (2009)

[2] A. A. Aguilar-Arevalo et al. [The MiniBooNE Collaboration], Phys. Rev. D81, 092005 (2010); A. A. Aguilar-Arevalo et al. [The MiniBooNE Collaboration], Phys. Rev. D81, 13005 (2010);A. A. Aguilar-Arevalo et al. [The MiniBooNE Collaboration], arXiv:1011.3572[hep-ex]; A. AguilarArevalo et al. [The MiniBooNE Collaboration], arXiv:1010.3264[hep-ex]; A. A. Aguilar-Arevalo et al. [The MiniBooNE Collaboration], Phys. Rev. D82, 092005 (2010); A. Aguilar-Arevalo et al. [The MiniBooNE Collaboration], Phys. Rev. Lett. 105, 181801 (2010); A. Aguilar-Arevalo et al. [The MiniBooNE Collaboration], Phys. Rev. Lett. 103, 111801 (2010);

[3] C. Athanassopoulos et al., Phys. Rev. C56 2806 (1997); R. B. Patterson, et al., NIM A608, 206 (2009)

[4] M. G. Catanesi et al. [The HARP Collaboration], Eur. Phys. J. C52, 29 (2007)

[5] Martini et al., Phys. Rev. C80, 065501 (2009) 